\title{
Additional Patient Factors may Predict Survival in Esophageal Cancer Better than TMN Alone After Esophagectomy
}

\author{
Johnathon Aho, MD, $\mathrm{PhD}^{\mathbf{1}}$, and Janani Reisenauer, $\mathrm{MD}^{\mathbf{1}}$ \\ Division of Thoracic Surgery, Mayo Clinic, Rochester, MN
}

Using the TMN classification alone to predict survival in patients with esophageal cancer has certain limitations, particularly for those who undergo esophagectomy. Prognostication, in esophageal cancer after resection, is critical to patient care. The predominant reason for this is the difficulty encountered in restaging patients after neoadjuvant therapy due to the profound inflammatory response from radiation. There also is variability in the treatment response observed among patients who undergo neoadjuvant therapy with a pathologic complete response reported in $29 \%$ of all comers and at least a partial response in $70 \%{ }^{1,2}$ Clearly, these factors are critical in determining overall survival (OS) of patients.

In this month's issue of Annals of Surgical Oncology, Deng and colleagues report a nomogram for predicting overall survival in esophageal cancer in a contemporary cohort. $^{3}$ Their results suggest that a nomogram model based on adjuvant therapy, sex, tumor location, grade, lymphovascular invasion, resected lymph nodes, and $\mathrm{T}$ and $\mathrm{N}$ stage is predictive of OS. This was then validated in an independent cohort, demonstrating a reasonable prognostic accuracy of $68 \%$ and $74 \%$ respectively by AUC analysis.

Only patients with squamous cell cancer were included in this study. When comparing the survival reported in the neoadjuvant chemoradiotherapy plus surgery versus surgery alone for oesophageal or junctional cancer (CROSS) trial for patients with squamous cell cancer, median overall survival was 81 months in the neoadjuvant chemoradiotherapy plus surgery group and 21 months in the surgery

(C) Society of Surgical Oncology 2019

First Received: 23 May 2019;

Published Online: 9 August 2019

J. Reisenauer, MD

e-mail: reisenauer.janani@mayo.edu alone group. ${ }^{4}$ This is in comparison to the current cohort, which excluded neoadjuvant therapy, but demonstrated a median survival of approximately 50 months, which is promising given the majority of patients were treated with surgery alone. These differences in survival may be due to several factors. Most importantly, those who underwent neoadjuvant therapy were excluded, whereas those who underwent adjuvant chemo radiotherapy were included. Both the training and validation cohorts for this model predominantly used surgery alone as a monotherapy: approximately $70 \%$. This limits the results of this study for generalization, because the majority (60\% or greater) of esophageal cancers have at least regional spread at the time of diagnosis. ${ }^{5}$ There have been demonstrable benefits of neoadjuvant chemoradiation therapy on survival, especially for those with regional disease. ${ }^{2}$

This study included only patients who were surgical candidates with adequate follow-up who survived the postoperative period. Prognostic data did align with what has been described previously. Those who underwent surgery alone had worse overall survival than those who received chemotherapy or chemoradiotherapy postoperatively. Additionally, this study corroborates the current literature that sex disparities, chemotherapy response, and the number of lymph nodes assessed affect survival. ${ }^{1,6,7}$ Furthermore, the majority mortalities within this population arose from local regional recurrence. This censoring is appropriate as patients who suffered perioperative mortality, and those who were lost to follow-up were not included in the study, because this would skew prognostic information.

The main impact of this work was that the authors were able to prognosticate overall survival by developing a scoring system and normogram, which performed favorably compared with the published AJCC survival curve using the TNM classification. ${ }^{8}$ Other nomogram models 
have been developed in Asian countries, in particular for squamous cell carcinoma; however, this was using a training model based on the SEER database, which notably is predominantly based on patients with predominantly adenocarcinoma. ${ }^{9}$ Cao et al. demonstrated that similar factors, such as age, race, histology, tumor site, tumor size, grade/depth of invasion, number of metastases, and resected nodes were independent prognostic factors for survival. $^{7}$ That model demonstrated reasonable prognostication improvements to TNM classification alone in both the training dataset, which was adenocarcinoma-predominant, and the Chinese validation cohort, which was predominantly squamous carcinoma. Disease models for survival after resection of esophageal cancer in the western world are significantly lacking for comparison. It would be interesting to know whether using the criteria from this nomogram would perform similarly for patients who undergo neoadjuvant treatment and resection of esophageal adenocarcinoma. Furthermore, it would be useful if these data perform similarly in patients with adenocarcinoma who received neoadjuvant therapy. Mortalities within the training and validation cohort were quite high, at 52\% and $51 \%$, respectively, but stage for stage did show survival prognosis based on their model.

We commend the authors for developing a reproducible normogram to predict overall survival for patients with squamous cell esophageal carcinoma. The authors have identified various factors that prognosticate overall survival and have validated their model. As the incidence of esophageal cancer increases, additional normograms will be required for patients who undergo multimodality therapy, for other histologic types of malignancy, and for patients who present with recurrence.

\section{REFERENCES}

1. Donahue JM, Nichols FC, Li Z, et al. Complete pathologic response after neoadjuvant chemoradiotherapy for esophageal cancer is associated with enhanced survival. Ann Thorac Surg. 2009;87(2):392-9. https://doi.org/10.1016/j.athoracsur.2008.11. 001

2. van Hagen P, Hulshof MCCM, van Lanschot JJB, et al. Preoperative chemoradiotherapy for esophageal or junctional cancer. $N$ Engl J Med. 2012;366(22):2074-84. https://doi.org/10.1056/nejm oa1112088

3. Deng W. Nomogram to predict overall survival in thoracic esophageal squamous cell carcinoma patients after radical esophagectomy. Ann Surg Oncol. (in press).

4. Shapiro J, Lanschot JJB van, Hulshof MCCM, et al. Neoadjuvant chemoradiotherapy plus surgery versus surgery alone for oesophageal or junctional cancer (CROSS): long-term results of a randomised controlled trial. Lancet Oncol. 2015;16(9):1090-8. https://doi.org/10.1016/s1470-2045(15)00040-6

5. Zhang Y. Epidemiology of esophageal cancer. World J Gastroenterol. 2013;19(34):5598-606. https://doi.org/10.3748/wjg.v19.i34. 5598

6. Baquet CR, Commiskey P, Mack K, Meltzer S, Mishra SI. Esophageal cancer epidemiology in blacks and whites: racial and gender disparities in incidence, mortality, survival rates and histology. J Natl Med Assoc. 2005;97(11):1471-8. https://www. pubmedcentral.nih.gov/articlerender.fcgi?artid=2594901\&tool= pmcentrez\&rendertype $=$ abstract. Accessed 4 Feb 2016.

7. Rowse PG, Jaroszewski DE, Thomas M, Harold K, Harmsen WS, Shen KR. Sex disparities after induction chemoradiotherapy and esophagogastrectomy for esophageal cancer. Ann Thorac Surg. 2017;104(4):1147-52. https://doi.org/10.1016/j.athoracsur.2017.05 .030

8. Rice TW, Patil DT, Blackstone EH. 8th edition AJCC/UICC staging of cancers of the esophagus and esophagogastric junction: application to clinical practice. Ann Cardiothorac Surg. 2017;6(2):119-30. https://doi.org/10.21037/acs.2017.03.14

9. Cao J, Yuan P, Wang L, et al. Clinical nomogram for predicting survival of esophageal cancer patients after esophagectomy. Sci Rep. 2016;6:26684. https://doi.org/10.1038/srep26684

Publisher's Note Springer Nature remains neutral with regard to jurisdictional claims in published maps and institutional affiliations. 